\title{
ZERTIFIZIERTE FORTBILDUNG
}

Folge 172

\section{Achtung:}

29_ Hyperthyreose im Alter

31_ Morbus Basedow

36_ CME-Fragen

1 1: $\ldots \ldots \ldots \ldots$

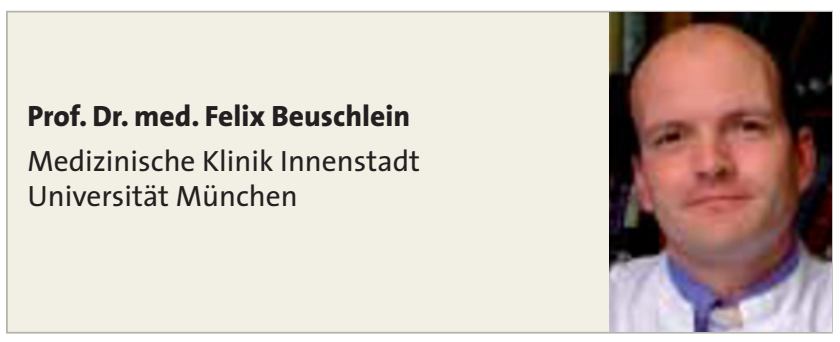

— Die Hyperthyreose ist eine relativ häufige Hormonstörung, deren adäquate Behandlung von der korrekten ätiologischen Zuordnung, der klinischen Ausprägung und den Begleiterkrankungen abhängt. Auch wenn Anamnese und klinische Untersuchung bei typischem Verlauf mit Tachykardie, Gewichtsabnahme, Hyperhidrosis und Durchfällen rasch die richtigen Hinweise geben, existieren dennoch diagnostische Fallstricke: Sie reichen von einer fehlenden, gering oder atypisch ausgeprägten klinischen Symptomatik (z.B. im höheren Lebensalter) über die bisweilen schwierige Interpretation von Laborbefunden in der Schwangerschaft oder bei schwerer Allgemeinerkrankung bis hin zu Problemen in der Differenzialdiagnostik. Die Besonderheiten im diagnostischen und therapeutischen Vorgehen bei der Hyperthyreose im Alter und der immunogenen Hyperthyreose behandeln beispielhaft die beiden folgenden Artikel.

\section{Subklinische Hyperthyreose - oft ein Zufallsbefund}

Nicht zuletzt durch die Einführung empfindlicher TSH-Assays der dritten Generation und deren häufigen Einsatz im Rahmen allgemeiner Screening-Untersuchungen nimmt allerdings auch die Zahl der zufällig entdeckten subklinischen Hyperthyreosen mit supprimiertem TSH und normalen peripheren Schilddrüsenparametern zu. So liegt in Deutschland der Anteil von Patienten mit einer subklinischen Hyperthyreose bei 1,8\% [1]. Ein nicht unerheblicher Teil dieser Patienten zeigt ein supprimiertes TSH im Rahmen einer (inadä- quat dosierten) Thyroxinsupplementierung. Ähnlich wie im Kollektiv der älteren Patienten mit Hyperthyreose sind die klinischen Manifestationen einer subklinischen Hyperthyreose in aller Regel unspezifisch oder nicht vorhanden. In Abhängigkeit von der Grunderkrankung (immunogene Hyperthyreose oder Autonomie in einer Struma nodosa) liegt die Wahrscheinlichkeit, eine manifeste Hyperthyreose zu entwickeln, pro Jahr zwischen 1 und $5 \%$. Sie ist umso höher, je niedriger der Ausgangs-TSH-Wert war [2].

Trotz meist geringer klinischer Ausprägung geht die Konstellation einer subklinischen Hyperthyreose mit einem erhöhten kardiovaskulären Risikoprofil und einer in manchen Studien gezeigten erhöhten kardiovaskulären Mortalität einher. Auch die Effekte einer latenten Hyperthyreose auf den Knochenstoffwechsel können sich bei Patienten ungünstig manifestieren [3]. Damit liegt ein eher aggressives Vorgehen auch zur Behandlung einer noch subklinischen Hyperthyreose nahe. Allerdings fehlen bisher prospektive Interventionsstudien, in denen klare Effekte der Normalisierung einer latenten Hyperthyreose auf harte Endpunkte wie kardiovaskuläre Mortalität oder Frakturrate gezeigt werden konnten [4]. In einer kürzlich publizierten Übersichtsarbeit [2] wird für asymptomatische Patienten mit subklinischer Hyperthyreose empfohlen, die Laborparameter nach drei bis sechs Monaten zu kontrollieren. Im Fall einer persistierenden TSH-Suppression soll eine differenzialdiagnostische Abklärung (Sonografie, ggf. Bestimmung der Schilddrüsenantikörper und Szintigrafie) erfolgen. In Abhängigkeit von Grunderkrankung und weiteren klinischen Befunden bzw. Risikofaktoren können dann Verlaufsbeobachtungen ausreichen (z. B. junge, asymptomatische Patienten) oder eine frühe sanierende Therapie (z.B. ältere Patienten mit Vorhofflimmern) angestrebt werden. Außerhalb der Indikationen einer TSH-suppressiven Behandlung von Schilddrüsenkarzinomen sollte die Induktion einer latenten Hyperthyreose durch eine Thyroxinsupplementierung durch adäquate Verlaufskontrollen und individuelle Dosisanpassung vermieden werden. 